\title{
Masked hypertension
}

\author{
Wilbert S. Aronow \\ Division of Cardiology, Department of Medicine, Westchester Medical Center and New York Medical College, Valhalla, NY, USA \\ Correspondence to: Wilbert S. Aronow, MD, FACC, FAHA. Division of Cardiology, Department of Medicine, Westchester Medical Center and New \\ York Medical College, Macy Pavilion, Room 141, Valhalla, NY 10595, USA. Email: wsaronow@aol.com.
}

Submitted Aug 21, 2017. Accepted for publication Sep 12, 2017.

doi: $10.21037 /$ atm.2017.09.24

View this article at: http://dx.doi.org/10.21037/atm.2017.09.24

Masked hypertension is diagnosed if the office blood pressure reading is not increased but the out-ofoffice-blood pressure reading by either home blood pressure monitoring or by ambulatory blood pressure monitoring is increased (1-3). Patients being treated for hypertension who have a normal office blood pressure reading but an increased out-of-office-blood pressure reading by either home blood pressure monitoring or by ambulatory blood pressure monitoring should be diagnosed as having masked uncontrolled hypertension (1). The European Society of Hypertension diagnosed an increased office blood pressure if the blood pressure was 140/90 mm Hg or higher and an increased outof-office-blood pressure if the home blood pressure reading was $135 / 85 \mathrm{mmHg}$ or higher, the daytime blood pressure reading measured by ambulatory blood pressure monitoring was $135 / 85 \mathrm{mmHg}$ or higher, or the 24 -hour ambulatory blood pressure reading was $130 / 80 \mathrm{mmHg}$ or higher (1). On the basis of current data, I would diagnose hypertension if the office systolic blood pressure was $130 \mathrm{mmHg}$ or higher or if the office diastolic blood pressure was $80 \mathrm{mmHg}$ or higher $(4,5)$.

Masked hypertension might be suspected in persons with an increased office blood pressure at some time, in young persons with normal or high normal office blood pressure who have left ventricular hypertrophy, in persons who have a family history of hypertension in both parents, in diabetics, in persons with multiple cardiovascular risk factors, and in obese individuals (1). Masked hypertension is estimated to occur in $10 \%$ to $30 \%$ of persons (1). Masked hypertension was diagnosed by ambulatory blood pressure monitoring in $14 \%$ of persons and by home blood pressure monitoring by $11 \%$ of persons (6). It may be reasonable to screen for masked hypertension with ambulatory blood pressure monitoring or with home blood pressure monitoring if the office systolic blood pressure is 120 to $129 \mathrm{mmHg}$ or if the office diastolic blood pressure is 75 to $79 \mathrm{mmHg}(6-10)$.

The prevalence of masked uncontrolled hypertension in 14,840 persons with treated hypertension in a Spanish registry was $31.1 \%$ (3). The prevalence of masked uncontrolled hypertension was higher in men, in persons with a borderline office blood pressure, and in smokers, diabetics, and obese persons (3). Of 5,007 persons with untreated hypertension, $8.1 \%$ had masked hypertension (6). Of 1,451 persons in this study with treated hypertension, $16.0 \%$ had masked uncontrolled hypertension. At 8.3-year median follow-up, cardiovascular events in those with untreated hypertension were increased $55 \%$ in persons with masked hypertension and 2.13 times in persons with sustained hypertension. Cardiovascular events in persons with treated hypertension were increased $76 \%$ in persons with masked hypertension and $40 \%$ in persons with uncontrolled hypertension (6).

Of 332 persons, 22\% had masked hypertension (7). Left ventricular mass index, carotid maximal intimamedia thickness, and urinary albumin level were higher in persons with masked hypertension than in persons with controlled hypertension and similar to those with sustained hypertension (7). A meta-analysis of seven studies including 11,502 persons, mean age 63 years, showed at 8.0-year follow-up that compared to normotensive persons, persons with masked hypertension had a 2.0 times increase in first cardiovascular events, and persons with sustained hypertension had a 2.28 times increase in first cardiovascular events (8).

A meta-analysis of eight studies including 7,964 persons showed that compared to normotensive persons, persons 
with masked hypertension had at follow-up a 2.09 times increase in cardiovascular events, and persons with sustained hypertension had a 2.59 times increase in cardiovascular events (9). In 8,237 untreated persons, mean age 50.7 years, in 12 population studies, the prevalence of masked hypertension ranged from $9.7 \%$ to $19.6 \%$ (10). At followup, compared to normotensive persons, persons with masked hypertension had a 1.76 to 2.03 times increase in cardiovascular events (10).

Of 1,332 Japanese persons, $16.6 \%$ had masked hypertension (11). At 10-year follow-up, compared to normotensive persons, persons with masked hypertension had a 1.88 times increased risk of cardiovascular disease mortality, a 2.17 times increased risk of stroke, and a 2.13 times increase in risk of cardiovascular disease mortality and stroke (11). Compared to normotensive persons, persons with sustained hypertension had a 1.94 times increased risk of cardiovascular disease mortality, a 2.83 times increased risk of stroke, and a 2.26 times increase in risk of cardiovascular disease mortality and stroke (11). Of 3,027 persons, mean age 47 years (50\% blacks), in the Dallas Heart Study, 17.8\% had masked hypertension (12). Persons with masked hypertension had increased aortic pulsed wave velocity, cystatin $\mathrm{C}$, and urinary albumin-to creatinine ratio. At 9.0-year median follow-up, after adjusting for traditional cardiovascular risk factors, compared with normotensive persons with masked hypertension had a 2.03 times increased risk of cardiovascular events, and persons with sustained hypertension had a 3.12 times increased risk of cardiovascular events (12).

In 972 blacks, mean age 60 years, in the Jackson Heart Study, masked hypertension was present in $25.9 \%$ of persons and in $34.4 \%$ of persons with a normal clinic blood pressure (13). Male gender, smoking, diabetes mellitus, and use of antihypertensive drugs were independent determinants of masked hypertension. Common carotid artery intima-media thickness, left ventricular mass index, and the urinary albumin to creatinine excretion ratio were higher in persons with masked hypertension than in persons with normal blood pressure and similar in persons with masked hypertension and with sustained hypertension (13). Fewer modifiable cardiovascular risk factors measured by the American Heart Association Life's Simple 7 was associated with a lower prevalence of masked hypertension in the Jackson Heart Study (14). In 644 participants in the Jackson Heart Study, the 10-year predicted atherosclerotic cardiovascular disease (ASCVD) risk was increased by masked hypertension 1.36 times by the pooled cohort risk equation if the ASCVD risk was 5\% to 7.4\%, 1.62 times if the ASCVD risk was $7.5 \%$ to $9.9 \%$, and 1.91 times if the ASCVD risk was $\geq 10 \%$ (15).

Of 5,636 Canadian persons with hypertension treated in a primary care setting, $19.6 \%$ and $36.8 \%$ of persons with controlled office blood pressure had masked uncontrolled hypertension (16). Diabetes mellitus, older age, male gender, obesity, and higher office systolic blood pressure were associated with masked uncontrolled hypertension (16). Of 803 persons, mean age 60 years, with treated and controlled office blood pressure, $32.1 \%$ had masked uncontrolled hypertension (17). Masked uncontrolled hypertension was due to increased nocturnal blood pressure in $22.3 \%$ of persons and to poor daytime blood pressure increase in $10.1 \%$ of persons. Masked uncontrolled hypertension was associated with male gender, longer duration of hypertension, obesity, smoking, and diabetes mellitus (17). Using data from 9,316 persons in the National Health and Nutrition Examination Survey 2005-2010, the estimated prevalence of masked hypertension in the United States was $12.3 \%$ of adults (18). Masked hypertension was higher in older persons, men, diabetics, and those with prehypertension (18).

In 813 participants, mean age 45 years, without treated hypertension in the Masked Hypertension Study, 15.2\% had masked hypertension (19). Echocardiographic left ventricular mass index was higher in persons with masked hypertension and in persons with prehypertension (19). In 652 Swiss persons, mean age 48 years, masked hypertension was present in $15.8 \%$ of persons (20). Masked hypertension was associated with age (odds ratio $=1.02$ ), high-normal office blood pressure (odds ratio $=6.68$ ), and with obesity (odds ratio $=3.63$ ) (20). In 588 Chinese patients with nondialysis chronic kidney disease, mean age 43 years, 20.6\% of patients had masked hypertension (21). At 35-month median follow-up, masked hypertension increased all-cause mortality 8.88 times, renal events 3.70 times, and major adverse cardiac and cerebrovascular events 8.66 times (21).

Nocturnal hypertension and non-dipping may be early markers of masked hypertension (22). Twenty-four hour ambulatory monitoring is the gold standard for diagnosing masked hypertension. Almost one-third of treated patients with masked hypertension have masked uncontrolled hypertension (22). There are scarce data on how to treat masked hypertension (23). One approach to treating masked hypertension would be to treat 24-hour ambulatory blood pressure (23). I would also aggressively treat modifiable 
risk factors for masked hypertension. Although I would treat masked hypertension because of its association with increased cardiovascular and cerebrovascular events and mortality, we do not have clinical trial data demonstrating that treating masked hypertension will reduce cardiovascular and cerebrovascular events and mortality. Since masked hypertension may be a prognostic marker of adverse cardiovascular and cerebrovascular events and mortality, clinical trials need to be performed to investigate whether masked hypertension should be treated with antihypertensive drug therapy.

\section{Acknowledgements}

None.

\section{Footnote}

Conflicts of Interest: The author has no conflicts of interest to declare.

\section{References}

1. O'Brien E, Parati G, Stergiou G, et al. European Society of Hypertension position paper on ambulatory blood pressure monitoring. J Hypertens 2013;31:1731-68.

2. Pickering TG, Davidson K, Gerin W, et al. Masked hypertension. Hypertension 2002;40:795-6.

3. Banegas JR, Ruilope LM, de la Sierra A, et al. High prevalence of masked uncontrolled hypertension in people with treated hypertension. Eur Heart J 2014;35:3304-12.

4. Wright JT Jr, Williamson JD, Whelton PK, et al. A randomized trial of intensive versus standard bloodpressure control. N Engl J Med 2015;373:2103-16.

5. Schiffrin EL, Calhoun DA, Flack JM. Do we need a new definition of hypertension after SPRINT? Am J Hypertens 2016;29:1127-9.

6. Stergiou GS, Asayama K, Thijs L, et al. Prognosis of white-coat and masked hypertension. International database of home blood pressure in relation to cardiovascular outcome. Hypertension 2014;63:675-82.

7. Tomiyama M, Horio T, Yoshii M, et al. Masked hypertension and target organ damage in treated hypertensive patients. Am J Hypertens 2006;19:880-6.

8. Fagard RH, Cornelissen VA. Incidence of cardiovascular events in white-coat, masked and sustained hypertension versus true normotension: a meta-analysis. J Hypertens 2007;25:2193-8.
9. Pierdomenico SD, Cuccurullo F. Prognostic value of white-coat and masked hypertension diagnosed by ambulatory monitoring in initially untreated subjects: an updated meta-analysis. Am J Hypertens 2011;24:52-8.

10. Asayama K, Thijs L, Li Y, et al. Setting thresholds to varying blood pressure monitoring intervals differentially affects risk estimates associated with white-coat and masked hypertension in the poulation. Hypertension 2014;64:935-42.

11. Ohkubo T, Kikuya M, Metoki H, et al. Prognosis of "masked" hypertension and "white-coat" hypertension detected by 24-h ambulatory blood pressure monitoring: 10-year follow-up from the Ohasama study. J Am Coll Cardiol 2005;46:508-15.

12. Tientcheu D, Ayers C, Das SR, et al. Target organ complications and cardiovascular events associated with masked hypertension and white-coat hypertension. Analysis from the Dallas Heart Study. J Am Coll Cardiol 2015;66:2159-69.

13. Diaz KM, Veerabhadrappa P, Brown MD, et al. Prevalence, determinants, and clinical significance of masked hypertension in a population-based sample of African-Americans: the Jackson Heart Study. Am J Hypertens 2015;28:900-8.

14. Bromfield SG, Shimbo D, Booth JN III, et al. Cadiovascular risk factors and masked hypertension. The Jackson Heart Study. Hypertension 2016;68:1475-82.

15. Ansley DE, Booth JN II, Abdalla M, et al. Predicted atherosclerotic cardiovascular disease risk and masked hypertension among blacks in the Jackson Heart Study. Circ Cardiovasc Qual Outcomes 2017;10:e003421.

16. Andalib A, Akhtari S, Rigal R, et al. Determinants of masked hypertension in hypertensive patients treated in a primary care setting. Intern Med J 2012;42:260-6.

17. Naser N, Dzubur A, Durak A, et al. Blood pressure control in hypertensive patients, cardiovascular risk profile and the prevalence of masked uncontrolled hypertension (MUCH). Med Arch 2016;70:274-9.

18. Wang YC, Shimbo D, Muntner P, et al. Prevalence of masked hypertension among US adults with nonelevated clinic blood pressure. Am J Epidemiol 2017;185:194-202.

19. Shimbo D, Newman JD, Schwartz JE. Masked hypertension and prehypertension: diagnostic overlap and interrelationships with left ventricular mass: the Masked Hypertension Study. Am J Hypertens 2012;25:664-71.

20. Alwan H, Pruijm M, Ponte B, et al. Epidemiology of masked and white-coat hypertension: the family-based SKIPOGH Study. PLoS One 2014;9: e92522. 
21. Wang C, Zhang J, Li Y, et al. Masked hypertension, rather than white-coat hypertension, has a prognostic role in patients with non-dialysis chronic kidney disease. Int J Cardiol 2017;230:33-9.

22. Franklin SS, O'Brien E, Staessen JA. Masked hypertension:

Cite this article as: Aronow WS. Masked hypertension. Ann Transl Med 2017;5(23):456. doi: 10.21037/atm.2017.09.24 understanding its complexity. Eur Heart J 2017;38:1112-8.

23. Peacock J, Diaz KM, Viera AJ, et al. Unmasking masked hypertension: prevalence, clinical implications, diagnosis, correlates and future directions. J Hum Hypertens 2014;28:521-8. 